\title{
Analysis on the Effect of Attack Skills and Tactics on Football Matches Scoring Efficiency
}

\author{
Yaoliang Zhang \\ Zhuhai College, Jilin University, Zhuhai, Guangdong, 519041 \\ hunter2011@foxmail.com
}

Keywords: Football Match; Skills and Tactics; Scoring Efficiency

\begin{abstract}
The use of statistical observation, comparative literature and other methods are summarized and analyzed the effects of different techniques and tactics of modern high-level football game shooting and scoring goals. The results show that a variety of scoring chances and goals with tactical obtained although still dominant, but misses the opportunity to transport control sudden, free kick, corner kick and other technical and tactical means were obtained and scored on the rise, especially in transport control sudden technical and tactical use of its growing role.
\end{abstract}

\section{Introduction}

Since UEFA EURO 1996, with the French team as the representative of technical football occupied the mainstream of world football in the past two European Championships and World Cup teams advocating technology we have made outstanding achievements, and follow the traditional with physical play as the main basis of the team subject to different levels of combat. The more typical German team, they invariably tactical style for decades been a serious challenge in the last five years, the results in the high level of competition in the world has repeatedly declined, its world-class team's position has also been shaken. German football in decline to give people a revelation: The core of football is the degree of the merits of individual players and the overall technical and tactical; physical fitness is the foundation and guarantee of technical and tactical; football technical and tactical factor in the race reflected the strength of both teams and disparities in levels, and different technical and tactical means to influence the game at a high level on the outcome of the game is different. So discussion of this issue will help in training and competition schedule targeted training content and layout of tactical play.

\section{Research Methods and Objects}

Observation statistics: observation and statistics 15th and the 16th World Cup total of 116 games for each team in the concrete application of different technical and tactical field goal in. Comparison of induction: the two-time World Cup statistics summarize, compare, and analyze the dynamic changes in the use of game. Literature: Check out this article discusses recent literature related to the content.

\section{Results and Discussion}

The Proportion of Different Technical and Tactical Way to Create Scoring Chances in the Game. World Cup shooting and scoring statistics show a different technical and tactical get scoring chances to score and the proportion is different. 16th statistical results before the World Cup 10 teams of different technical and tactical means to get scoring chances and goals indicate, get scoring opportunities with technical and tactical means to get the most scoring chances, the total number of shots of $5416 \%$, incident The ball is also up, reaching 64 goals, scoring $6217 \%$ of the total number. This figure shows that the most important way to get a shot of modern football game opportunities through a variety of means, ranging from a variety of regional and obtained the number of tactical coordination, which is the first four teams in the most obvious. World champions France in the World Cup 7 games scoring chances were obtained 157 times, an average of 2214 times per game 
shot, which misses the opportunity to get through a variety of complex 91 times, 58\% of its total, higher than the average of the previous 10 teams, by blending 5 goals, accounting for $3313 \%$ of the total number of goals. French team to make it through organized with the technical and tactical play in a variety of opponents in the game can get very smoothly implemented. This is based on the French team have a strong midfield support, Zinedine Zidane, Didier Deschamps combination of tactics, Djorkaeff, Thierry Henry, Marcel Desailly and other frontcourt players have played a considerable power in the attack, whether it is Road or sidewalk and rib tactical coordination, both continue to provide adequate conditions for the French team to score goals. The performance of the more prominent by Brazil, the Netherlands, Croatia, Argentina and Denmark and other teams in this regard. Brazil through to get scoring chances, although with not dominant, but it was the first shot on goal 10 exclusive first team, scoring 11 goals, $7816 \%$ of the total number of goals. Followed by the Netherlands team, the team corresponding figure is seven games with a shot 82 times, fired 10 goals, respectively, the total number of shots and the total number of goals $55 \%$ and $7619 \%$. These two teams always pay attention to the style of tactical coordination, get scoring opportunities through cooperation and achieve goals aimed at Brazil and the Netherlands has always pursued the policy. The first 10 on the list of several teams also presented in this state, therefore, misses the chance to get through with the fight for a high level of football competition is the most important modern ways and means.

Win Control sudden technical and tactical use in the game is another important means of obtaining scoring chances and goals, shots and goals of its total $2517 \%$, respectively, and $1018 \%$, this aspect of the performance of the most prominent is the French team and the Netherlands team. France won by technical and tactical transport control projection 42 shots, fired into 4 balls, accounting for $2618 \%$ and $2617 \%$ of the total number of misses and goals are; the Netherlands corresponding figures are shipped sudden control of 35 shots, He fired three goals, respectively, accounting for $2315 \%$ and $2311 \%$ of the total number of misses. In this regard the team as well as the outstanding performance of Argentina and Nigeria, the two scoring chances detachment obtained by such means is more, accounted for $33 \%$ and $4117 \%$, respectively, the total number of teams a shot, but unfortunately did not score. The importance of technical and tactical transport control projection in modern football matches has been growing attention in the world are particularly high level of attention to the number of teams to arrange transportation, ranging from a strong ability to control sudden players in the lineup is set, these players tend to open up the situation at a critical time, to lay the foundation for the tournament victory. Serving free kick and corner kick in the game growing, but its total impact on the game can not shake with the sudden and transport control technical and tactical position in the race, but both the technical and tactical means of scoring rate to up with the technical and tactical transport control projection scoring rate. As can be seen from Table 1, the first 10 teams overall scoring rate kicks and corners all over the complex and transport control sudden, the former scoring rate was 1316,1712, the latter scoring rate was 413, 1216. It is especially high corner of the amazing scoring rate is not already, the French team is still ahead in this regard, seven games to get 14 shots through the corner, into the 5 ball, which is never in the calendar year in the World Cup before, the French team in the final to beat Brazil 3B0, which has two goals is through the corner of the feed for the final victory of the game, for the French team won the world championship for the first time established a Tougong.

The Prominent Role of Transport Control Shock Tactics in the Game. A striking feature of the past two World Cup athletes appearing in the game frequently use technical and tactical transport control projection provide and create opportunities for the shot misses and direct implementation. Mainly from the 15th and the 16th World Cup goal misses the point of view of technical means can be clearly demonstrated this (Table 1).

Data shown in Table 1, although a direct shot on goal technology still occupy the dominant position, but four years between the 15th to the 16th World Cup World Cup, the modern high-level football game Shooting the use of technology has occurred several change, has served as the dominant technology in direct radio has shown a downward trend, while the transport control sudden shot, then shot trip, head shot, etc. uptrend, especially transport control projection 
technology by the $2418 \%$ increase in the total number of goals to $2715 \%$, goals also increased, indicating that this misses the technology more prominent role in the game. This change in the past 10 years the development of football offensive and defensive tactics are very closely linked, more and more attention to defense balance of modern football, the defense in the backcourt presents multi-level, three-dimensional, network-based defense system, which the attackers to say, get scoring chances and goals more difficult, heavily guarded frontier closed area in the implementation of a direct shot very difficult, especially between two evenly matched teams in the game, to score through direct shot more difficult. In the more considerable strength in the game, is not a simple field goal by 1 to 2 technical movements, with 1 to 2 kinds of tactics can be successful, but to continuously repeated and varied use of a variety of technical and tactical action, will it be possible to create opportunities to score, transport control projection technology in which it plays an important role. Support for the two-time World Cup scoring last pass before the Technical Usage statistics proved this point.

Table 1 Goals last pass before support the use of statistical techniques

\begin{tabular}{|l|l|l|l|l|l|l|l|}
\hline Times & Scoring rate & Dribble & Direct shot & Indirect shot & Head shot & Own goals & Total \\
\hline \multirow{2}{*}{ 15th } & \multirow{2}{*}{ Ratio score \% } & 35 & 64 & 18 & 23 & 1 & 141 \\
\cline { 3 - 8 } & & 24.8 & 45.4 & 12.8 & 16.3 & 0.7 & \\
\hline \multirow{2}{*}{36.8} & \multirow{2}{*}{ Ratio score \% } & 47 & 63 & 27 & 31 & 3 & 171 \\
\cline { 3 - 7 } & & 27.5 & 36.8 & 15.8 & 18.1 & 1.8 & \\
\hline
\end{tabular}

Field goal before the last pass for the quality of the shot has a very important role, and support for the last time before scoring pass to use different techniques for improving the accuracy of passing and timing is crucial to grasp the ball. From the two World Cup matches, the sudden mass transport controls the largest proportion. Over the chances of such a high level in the World Cup soccer tournament, wanted to attack a pass or be able to successfully break into the restricted area directly to the companion is very small, a large number of breakthrough success and get scoring chances.

\section{Conclusion}

Our football team at all levels should correctly understand the impact of different technical and tactical field goal, on the basis of traditional importance to cooperation based on a variety of technical and tactical means to improve the ability of other forms of offensive way, we make an effort to research and develop positioning the ball tactics program, trained expertise free kick, corner including special players in the vicinity of the bottom line of the throw. Training team members have had to establish a strong sense of desire and breakthrough thinking, today's football game against the essence is the high level of competition for the ball, who mastered a long time possession of the initiative will be able to master the game, so from footballer Enlightenment stage should inculcate this idea, and in each training can be reflected in both, the technical and tactical and mass transport control, then, radio technology and personal, local and global tactics combined and integrated to improve their transport sudden ability to control and use levels. We should vigorously promote the flamboyant our football players, football players change our commonalities too strong, lack of personality weaknesses, advocating soccer players in the competition resourcefulness and improvisation, train athletes innovation and innovative ability in training game.

\section{References}

[1] Wei-Ri R. 141 Goals for Technical and Tactical Characteristics of the Study. China Sports Science, 1995, (11): 30-33.

[2] Yang-Yi M. 1998 World Technology Trends. 16th World Cup Album. Chinese Football Association, 1999, 13-6.

[3] Huang-Xian W. Dribbles from the Use of Technology to See the Development of Football Technique. China Sports Science and Technology, 1995 (11): 34-36. 